\title{
SMA Based Actuators for Pressure Ulcer Prevention
}

\author{
Ricardo Carvalho ${ }^{1}$, Joana Almeida ${ }^{1}$, Pedro Pereira ${ }^{1}$, Susana Pereira ${ }^{2}$, Ricardo Simoes ${ }^{2}$, Miguel Ribeiro ${ }^{1}$, \\ Joana Fonseca ${ }^{1}$ \\ ${ }^{1}$ CeNTI - Centre for Nanotechnology and Smart Materials \\ Rua Fernando Mesquita 2785, 4760-034 Vila Nova de Famalicão, Portugal \\ rcarvalho@centi.pt; jalmeida@centi.pt; pmpereira@centi.pt; mribeiro@centi.pt; jfonseca@centi.pt \\ ${ }^{2}$ IPCA - Polytechnic Institute of Cávado and Ave \\ Vila Frescaínha S. Martinho, 4750-810 Barcelos, Portugal \\ spereira@ipca.pt; rsimoes@ipca.pt
}

\section{Extended Abstract}

Loss of voluntary limb movement due to chronic degenerative diseases, particularly in the elderly population, road accidents, and other circumstances, can lead to the immobilization of a person that may result in the development of pressure ulcers. These ulcers result primarily from the compression of soft tissue between a bone prominence and an external surface for an extended period and may involve different types of tissue, muscle, and bone [1], [2]. Aiming the reduction and prevention of pressure ulcers in bedridden patients, there are different commercial solutions. The most common are passive systems such as viscoelastic memory foam mattress (able to relieve pressure points by adapting it to body shape) or active solutions such as pressure air alternating systems (able to dynamically alternate pressure between two or three body regions). More recently, some of these active systems already include a sensing system to monitor pressure in critical regions [3], [4] and [5]. Usually, they are limited to partial regions of alternate actuation. Furthermore, the use of air systems implies the presence of a compressor pump, which causes noise and discomfort during its operation.

Here we present a novel actuation solution for an intelligent relieving pressure system, which aims the reduction and prevention of pressure ulcers in bedridden patients. In a real-time mode, the proposed solution identifies regions where the pressure is above critical values and will actuate providing pressure relief. As an alternative to the widely used actuation mechanism in commercial systems, new actuating mechanisms based on Shape Memory Alloys (SMAs) were engineered.

SMAs are a unique class of smart materials with the ability to return to their original shape when heated after several deformations. These alloys can be subject to a memorization process and be deformed at low temperatures (martensite), presenting a pseudo-plastic behavior. SMAs will stay deformed until the temperature increases (austenite), whereupon they will return to their memorized shape. These alloys can be used as both sensors and actuators due to their compactness, lightness, bio-compatibility, noiseless operation and high wear resistance providing significant strokes and forces [6].

The proposed actuation mechanism has an innovative design combining high performance, rigidity, and lightness. It consists on a set of platforms with reduced dimensions, coupled to SMAs springs. Applying an electric voltage to the SMA springs, their temperature raises until phase transition resulting in a vertical movement of the actuator. The use of SMAs in the form of a spring allows obtaining substantially higher displacements in comparison with the use of straight SMA wire. This feature contributes to the compactness of the final actuators.

Based on the innovative designs, several prototypes were constructed using 3D printing and actuated by NiTi springs with a transition temperature of $80{ }^{\circ} \mathrm{C}$, which were mechanical, electrical and thermally characterized. The most promising solution has displayed an actuation force of $30 \mathrm{~N}$ during an actuation period of $15 \mathrm{~s}$.

\section{References}

[1] "Pressure ulcers prevalence, cost and risk assessment: consensus development conference statement--The National Pressure Ulcer Advisory Panel.," Decubitus, vol. 2, no. 2, pp. 24-8, May 1989.

[2] J. Bridel, "The aetiology of pressure sores," J. Wound Care, vol. 2, no. 4, pp. 230-238, Jul. 1993.

[3] AlertaMedical, Making cutting-edge medical equipment affordable, 2018. [Online]. Available: https://www.alertamedical.com/en [21 May, 2018]. 
[4] Malvestio. (2018). Furniture For Healthcare Facilities [Online]. Available: https://malvestio.it/en/ [21 May, 2018 ].

[5] S. Med. (2018). UP AIR [Online]. Available: https://www.servicemed.it/en/products/up-air/ [22 May, 2018].

[6] J. Mohd Jani, M. Leary, A. Subic, and M. A. Gibson, "A review of shape memory alloy research, applications and opportunities,” Mater. Des., vol. 56, pp. 1078-1113, Apr. 2014. 